\title{
Prevalence and profiles of ramucirumab-associated severe ascites in patients with hepatocellular carcinoma
}

\author{
NAOKI KAMACHI ${ }^{1}$, SHIGEO SHIMOSE ${ }^{1}$, KEISUKE HIROTA ${ }^{2}$, SHUNJI KOYA ${ }^{2}$, \\ HIDEKI IWAMOTO ${ }^{1}$, TAKASHI NIIZEKI ${ }^{1}$, TOMOTAKE SHIRONO ${ }^{1}$, \\ MASAHITO NAKANO ${ }^{1}$, RYUKI HASHIDA ${ }^{2,3}$, TAKUMI KAWAGUCHI ${ }^{1}$, HIROO MATUSE ${ }^{2}$, \\ KAZUNORI NOGUCHI $^{4}$, HIRONORI KOGA ${ }^{1}$ and TAKUJI TORIMURA ${ }^{1}$
}

\begin{abstract}
${ }^{1}$ Division of Gastroenterology, Department of Medicine, Kurume University School of Medicine; ${ }^{2}$ Division of Rehabilitation, Kurume University Hospital; ${ }^{3}$ Department of Orthopedics, Kurume University School of Medicine, Kurume, Fukuoka 830-0011; ${ }^{4}$ Department of Gastroenterology and Hepatology, Omuta City Hospital, Omuta, Fukuoka 832-0077, Japan
\end{abstract}

Received November 16, 2020; Accepted February 12, 2021

DOI: $10.3892 /$ mco.2021.2241

\begin{abstract}
Severe ascites is an adverse event of ramucirumab (RAM), a second-line treatment for hepatocellular carcinoma (HCC). Ascites can be associated with various factors, including nutritional status and muscle quality. The aim of the present study was to investigate the prevalence and profiles of RAM-associated severe ascites in patients with HCC. This retrospective study enrolled 14 consecutive patients with HCC treated with RAM (median age, 72 years; Barcelona Clinic Liver Cancer stage B/C, 6/8). Nutritional status and muscle quality were evaluated using the controlling nutritional status (CONUT) score and intramuscular adipose tissue (IMAT) content, respectively. Factors associated with severe ascites were evaluated using decision-tree analysis. The median progression-free survival (PFS) time was 2.1 months, and the overall objective response and disease control rates were 14 and $50 \%$, respectively. Severe ascites developed in $57.1 \%$ of the patients, and the median onset was 37.5 days (range, 14-61 days) after initiation of RAM treatment. In the decision-tree analysis, the CONUT score and IMAT content were the first and second splitting variables for the development of severe ascites. In patients with a CONUT score $\geq 5$ and IMAT $<-0.54$, the prevalence of severe ascites was
\end{abstract}

Correspondence to: Dr Shigeo Shimose, Division of Gastroenterology, Department of Medicine, Kurume University School of Medicine, 67 Asahi-machi, Kurume, Fukuoka 830-0011, Japan E-mail: shimose_shigeo@med.kurume-u.ac.jp

Abbreviations: HCC, hepatocellular carcinoma; MTA, molecular targeted agent; RAM, ramucirumab; AFP, $\alpha$-fetoprotein; VEGF; vascular endothelial growth factor; PFS, progression-free survival; CONUT, Controlling Nutritional Status; IMAT, intramuscular adipose tissue; ALBI, albumin-bilirubin; CT, computed tomography; $\mathrm{AE}$, adverse event; LEN, lenvatinib

Key words: AE, hepatoma, malnutrition, RAM, severe ascites
80 and $100 \%$, respectively. A high incidence of severe ascites was observed in patients treated with RAM. A CONUT score $\geq 5$ and an IMAT $<-0.54$ were associated with severe ascites. Thus, caution must be taken for severe ascites in patients with HCC treated with RAM, in particular patients with malnutrition and fat infiltration in muscle.

\section{Introduction}

Hepatocellular carcinoma (HCC) is a common malignancy and one of the major leading causes of cancer deaths worldwide (1) Patients with unresectable HCC show poor prognoses (2) Systemic chemotherapy is a treatment strategy for unresectable HCC. Molecular targeted agents (MTAs), such as sorafenib (SORA), regorafenib (REGO), and lenvatinib (LEN), are available for the treatment of unresectable HCC (3-5). Ramucirumab (RAM), an MTA, has recently been approved for second-line treatment of unresectable HCC patients with a baseline $\alpha$-fetoprotein (AFP) concentration $\geq 400 \mathrm{ng} / \mathrm{ml}(6)$.

RAM is a human immunoglobulin G1 (IgG1) monoclonal antibody that inhibits ligand activation of vascular endothelial growth factor (VEGF) receptor 2. In a phase 3 trial (REACH-2 trial) involving unresectable HCC patients, RAM significantly improved progression-free survival (PFS) and overall survival relative to placebo (6) However, in the REACH-1 trial, more than $5 \%$ of the patients treated with RAM suffered grade $\geq 3$ treatment-emergent adverse events (AEs), including ascites. Moreover, according to the REACH-2 trial, patients with Child-Pugh scores of 7 and 8 showed a higher incidence of RAM-related grade 3 ascites than those with Child-Pugh scores of 5 or $6(7)$.

The development of ascites in patients with HCC is associated with various factors, including malnutrition (8) The controlling nutritional status (CONUT) score is a nutritional index consisting of three parameters: Serum albumin level, total cholesterol level, and lymphocyte count (9) CONUT is superior than other nutritional assessment tools such as the Nutrition Risk Screening-2002 and subjective global assessment to predict infectious complication in patients with 
digestive diseases (10). In addition, the CONUT score was reported to predict the development of ascites in patients with HCC (11) While, intramuscular adipose tissue (IMAT) content is a method for the quantification of fatty infiltration in skeletal muscles (12) IMAT is reported to be an independent risk factor for mortality in patients with HCC (13) IMAT was also reported to be significantly associated with liver dysfunction and the prognosis of HCC patients who underwent hepatectomy (14).

The aim of this study was to investigate patient profiles, including the CONUT score and IMAT content, associated with the development of RAM-related ascites in patients with HCC.

\section{Materials and methods}

Study design. This retrospective study was conducted at the Kurume University Hospital and Omuta City Hospital. The protocol conformed to the ethical guidelines of the 1975 Declaration of Helsinki and was approved by the ethics committees of the Kurume University School of Medicine (approval no. 19203). An opt-out approach was employed to obtain informed consent from the patients, and personal information was protected during data collection.

Inclusion and exclusion criteria. The patient inclusion criteria for this study were as follows: i) diagnosis of unresectable HCC according to the Barcelona Clinic Liver Cancer (BCLC) staging system (15); ii) age $\geq 18$ years; iii) Eastern Cooperative Oncology Group performance status 0 ; iv) history of pretreatment with MTAs; and v) completion of follow-up until death or study cessation (August 30, 2020). The patient exclusion criteria were as follows: i) history of a malignant tumor other than $\mathrm{HCC}$ in the 5 years preceding the study; ii) participation in any clinical trial; iii) Child-Pugh class $\mathrm{C}$; iv) creatinine $>1.5 \mathrm{mg} / \mathrm{dl}$; v) chronic heart failure; vi) infiltrative HCC; vii) presence of ascites; viii) esophageal varices with a high risk of rupture; and ix) history of liver transplantation.

Patients. A total of 16 consecutive HCC patients who received RAM treatment between September 19, 2019 and July 31, 2020 were registered. The data cutoff date for this analysis was August 31, 2020. Patients meeting any of the exclusion criteria were excluded from the analysis $(n=2)$. Thus, a total of 14 patients were enrolled in the study.

Assessment of nutritional status and liver function. CONUT score, used to assess nutritional status, was calculated from serum albumin level, total cholesterol level, and lymphocyte count, as previously described (9) Albumin concentrations of $\geq 3.5,3.0-3.49,2.5-2.99$, and $<2.5 \mathrm{~g} / \mathrm{dl}$ were scored as 0, 2, 4 and 6 points, respectively. Total lymphocyte counts of $\geq 1,600,1,200-1,599,800-1,199$, and $<800 / \mu 1$ were scored as $0,1,2$ and 3 points, respectively. Total cholesterol concentrations of $\geq 180,140-179,100-139$ and $<100 \mathrm{mg} / \mathrm{dl}$ were scored as 0, 1, 2 and 3 points, respectively. Liver function was evaluated using the albumin-bilirubin (ALBI) score, as previously described (16) It based on serum albumin and total bilirubin levels; ALBI-score $=[\log 10$ bilirubin $(\mu \mathrm{mol}$ /l) $\mathrm{x}$ 0.66] + [albumin $(\mathrm{g} / \mathrm{l}) \mathrm{x}-0.085]$, and was graded as following: $\leq-2.60=$ ALBI grade $1,>-2.60$ to $\leq-1.39=$ ALBI grade $2,>-1.39=$ ALBI grade 3 ).
Evaluation of skeletal muscle index. The skeletal muscle index (SMI) was evaluated at the third lumbar vertebra level on computed tomography (CT) scans, which were obtained as part of the HCC assessment (17) SMI were calculated by normalizing the L3 skeletal muscle areas by the square of the height $\left(\mathrm{m}^{2}\right)(18)$. The targets of measurement were psoas, erector spinae, quadratus lumborum, transversus abdominis, external and internal obliques, and rectus abdominis. The measurement was performed by two government-certified physical therapists (S.K. and K.H.) who were blinded to the patients' information. This analysis was performed using diagnostic software Image J Version 1.50 software (National Institutes of Health) (19).

Evaluation of visceral fat area (VFA). We measured VFA using diagnostic CT scans at the umbilical level, as previously described (20) CT scanning was performed for HCC evaluation. The measurement was performed by two government-certified physical therapists (S.K. and K.H.) who were blinded to the patients' information. The VFA was also measured by the diagnostic software ImageJ Version 1.50 software (National Institutes of Health) (19).

Evaluation of IMAT. Muscle quality was evaluated by measuring the IMAT content. The IMAT was calculated as the psoas muscle-to-subcutaneous fat attenuation ratio using diagnostic CT scans at the umbilical level as previously described (21) A higher IMAT indicates a greater amount of adipose tissue within the skeletal muscle (22) The SMI, VFA, and IMAT were measured by two government-certified physical therapists (S.K. and K.H.) who were blinded to patient information.

Diagnosis of HCC. HCC was diagnosed using a combination of tests for serum tumor markers, such as AFP and des- $\gamma$-carboxy prothrombin (DCP), and imaging procedures, such as ultrasonography, computed tomography (CT), and magnetic resonance imaging (MRI). HCC was classified using the BCLC staging system (15).

Treatment with RAM and evaluation of therapeutic response. RAM was intravenously injected at a dose of $8 \mathrm{mg} / \mathrm{kg}$ once every 2 weeks. Therapeutic response was evaluated according to the modified Response Evaluation Criteria in Solid Tumors (mRECIST) (23), using dynamic CT or magnetic resonance imaging. The evaluation was conducted 4-6 weeks after initiation of treatment with RAM, and thereafter, at intervals of 2-3 months until death or study cessation.

Assessment of AEs and ascites. AEs and ascites were assessed every month after the initiation of RAM treatment. AEs were assessed according to the National Cancer Institute Common Terminology Criteria for Adverse Events, version 4.0.

Definition of severe ascites. Ascites were assessed by ultrasonography and CT scan images. Severe ascites were defined as diuretic-resistant ascites.

Statistical analysis. All data are expressed as frequency or median (range). All statistical analyses were performed using 
Table I. Clinicopathological characteristics of patients with hepatocellular carcinoma $(n=14)$.

\begin{tabular}{|c|c|}
\hline Characteristic & Value \\
\hline Median age (range), years & $72.5(36-87)$ \\
\hline Sex, female/male & $4 / 10$ \\
\hline Median body mass index (range) & $20.7(16.4-24.2)$ \\
\hline Etiology, HBV/HCV/others & $4 / 5 / 5$ \\
\hline Child-Pugh class, A/B & $11 / 3$ \\
\hline Median total cholesterol (range), mg/dl & $166(135-254)$ \\
\hline Median ALBI score (range) & $-2.19(-2.74--1.65)$ \\
\hline Modified ALBI grade, $1 / 2 \mathrm{a} / 2 \mathrm{~b} / 3$ & $1 / 6 / 7 / 0$ \\
\hline \multicolumn{2}{|l|}{ Controlling nutritional status score } \\
\hline $0-1$ (normal) & 3 \\
\hline 2-4 (mild) & 6 \\
\hline $5-8$ (moderate) & 5 \\
\hline$\geq 9$ (severe) & 0 \\
\hline Median tumor diameter (range), mm & $47.8(11.0-132.0)$ \\
\hline Number of tumors, $<5 / \geq 5$ & $1 / 13$ \\
\hline Barcelona clinic liver cancer stage, B/C & $6 / 8$ \\
\hline Macroscopic portal vein invasion, yes/no & $2 / 12$ \\
\hline Extrahepatic metastasis, yes/no & $8 / 6$ \\
\hline Median $\alpha$-fetoprotein (range), $\mathrm{ng} / \mathrm{ml}$ & $5,917(906-612,770)$ \\
\hline Median des- $\gamma$-carboxy prothrombin (range), $\mathrm{mAU} / \mathrm{ml}$ & $2,035(22-292,689)$ \\
\hline Intramuscular adipose tissue & $-0.51(-0.72--0.38)$ \\
\hline Median visceral fat mass (range), $\mathrm{cm}^{2}$ & $79.92(24.5-162.3)$ \\
\hline Muscle atrophy, yes/no & $11 / 5$ \\
\hline Prior treatment with molecular targeted agents, SORA/SORA+LEN/LEN & $4 / 5 / 5$ \\
\hline Diuretic, $+/-$ & $8 / 6$ \\
\hline Median follow-up duration (range), months & $4.5(1.3-11.5)$ \\
\hline
\end{tabular}

HBV, hepatitis B virus; HCV, hepatitis C virus; ALBI, albumin-bilirubin; SORA; sorafenib; LEN, lenvatinib.

JMP Pro, version 14 (SAS Institute Inc.). PFS was calculated using the Kaplan-Meier method and analyzed using the log-rank test. We also performed decision tree analysis to identify factors associated with severe ascites, as previously described (24).

\section{Results}

Patient characteristics. Patient profiles are summarized in Table I. The median patient age was 72.5 years, and $28.5 \%$ of the patients were female. The median body mass index (BMI) was $20.7 \mathrm{~kg} / \mathrm{m}^{2}$, and $78.6 \%$ of patients $(11 / 14)$ showed Child-Pugh class A. ALBI grade 2 was observed in $92.3 \%$ of the patients, and ALBI 2 a was observed in $42.9 \%$. The frequencies of CONUT scores of 0-1 (normal nutrition), 2-4 (mild malnutrition), 5-8 (moderate malnutrition), and $\geq 9$ (severe malnutrition) were $21.4,42.9,35.7$ and $0.0 \%$, respectively (Table I). BCLC stage B HCC was seen in $42.8 \%$ of the patients. The median IMAT and VFA were -0.51 and $79.92 \mathrm{~cm}^{2}$, respectively. Prior treatment with sorafenib (SORA), SORA+LEN, and LEN was seen in $28.6(4 / 14), 35.7(5 / 14)$, and $35.7 \%(5 / 14)$ of the patients, respectively. The median observation period was 4.5 months (1.3-11.5 months) (Table I).
Table II. Treatment response rate to ramucirumab in patients with hepatocellular carcinoma $(n=14)$.

\begin{tabular}{ll}
\hline Response & $\mathrm{N}(\%)$ \\
\hline Complete response & $0(0)$ \\
Partial response & $2(14)$ \\
Stable disease & $5(36)$ \\
Progressive disease & $7(50)$ \\
Objective response rate & $2(14)$ \\
Disease control rate & $7(50)$ \\
\hline
\end{tabular}

Evaluation of treatment response and PFS. The therapeutic responses to RAM are shown in Table II. Complete response, partial response, stable disease, and progressive disease were observed in $0,14,36$ and $50 \%$ of the patients, respectively (Table II). The overall objective response rate and disease control rate were 14 and $50 \%$, respectively (Table II). The median PFS time was 2.1 months (Fig. 1).

AEs of RAM. AEs determined by the attending physician are shown in Table III. Ascites was the most frequent AE and was 
Table III. Adverse events associated with ramucirumab treatment in patients with hepatocellular carcinoma $(\mathrm{n}=14)$.

\begin{tabular}{lcccc}
\hline Adverse event & Any, $\mathrm{n}(\%)$ & Grade $1, \mathrm{n}(\%)$ & Grade 2, $\mathrm{n}(\%)$ & Grade $\geq 3, \mathrm{n}(\%)$ \\
\hline Ascites & $8(57.1)$ & $0(0.0)$ & $2(14.2)$ & $6(42.9)$ \\
Appetite loss & $7(50.0)$ & $3(21.4)$ & $3(21.4)$ & $1(7.2)$ \\
Fatigue & $7(50.0)$ & $4(28.6)$ & $3(21.4)$ & $0(0.0)$ \\
Hypertension & $5(35.6)$ & $3(21.4)$ & $2(14.2)$ & $0(0.0)$ \\
Diarrhea & $5(35.6)$ & $3(21.4)$ & $2(14.2)$ & $0(0.0)$ \\
Proteinuria & $4(28.6)$ & $1(7.2)$ & $3(21.4)$ & $0(0.0)$ \\
Hand-foot-skin-reaction & $0(0.0)$ & $0(0.0)$ & $0(0.0)$ & $0(0.0)$ \\
Infusion reaction & $1(7.2)$ & $0(0.0)$ & $1(7.2)$ & $0(0.0)$ \\
\hline
\end{tabular}

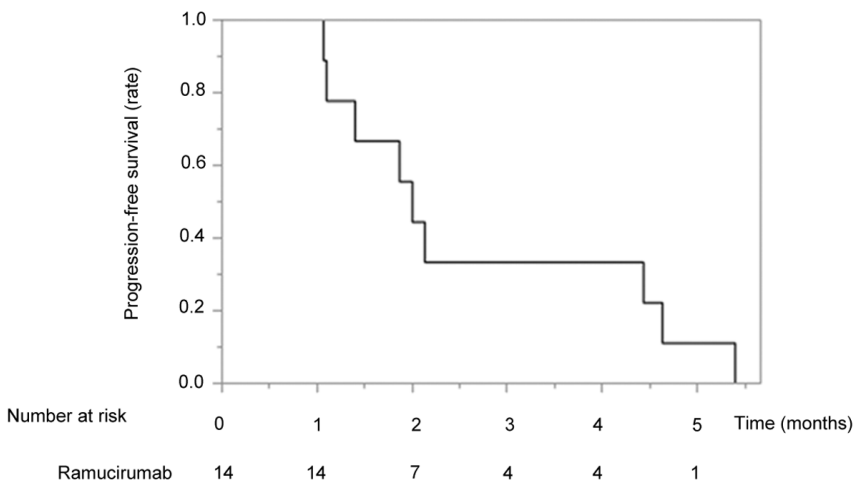

Figure 1. Kaplan-Meier curve for progression-free survival of patients with hepatocellular carcinoma treated with ramucirumab $(n=14)$.

seen in $57.1 \%(8 / 14)$. Appetite loss and fatigue were seen in $50(7 / 14)$ and $50 \%(7 / 14)$, respectively. The infusion reaction was seen in $7.2 \%(1 / 14)$.

Prevalence of RAM-related severe ascites. RAM-related severe ascites developed in $57.1 \%(8 / 14)$ of the patients, and the median onset was 37.5 days (14-61 days) after RAM treatment initiation. Although ascites was treated with diuretics, RAM was discontinued in $87.5 \%$ (7/8) of patients who developed severe ascites. A representative case of the development of severe ascites treated with RAM is shown in Fig. 2A and B. Visceral inversion was seen in this case. No ascites was seen when multiple recurrent hepatic nodules developed after LEN treatment (Fig. 2A). Severe ascites developed 27 days after the initiation of treatment with RAM (Fig. 2B).

Decision-tree analysis for RAM-related severe ascites. In this study, severe ascites developed in $57 \%$ of all the subjects by the time of study cessation. To determine the profiles associated with RAM-related severe ascites, decision tree analysis was performed. We revealed that the CONUT score was the first splitting variable for the development of RMA-related severe ascites. In patients with a CONUT score $<5$, the second splitting variable was the IMAT (Fig. 2). Although severe ascites developed in all patients with an IMAT $<-0.54$ and a CONUT score $<5$, severe ascites developed in only $16 \%$ of patients with an IMAT $\geq-0.54$ and a CONUT score $<5$ (Fig. 3).

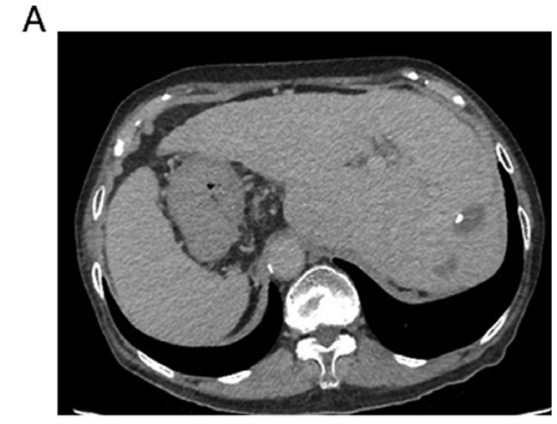

B

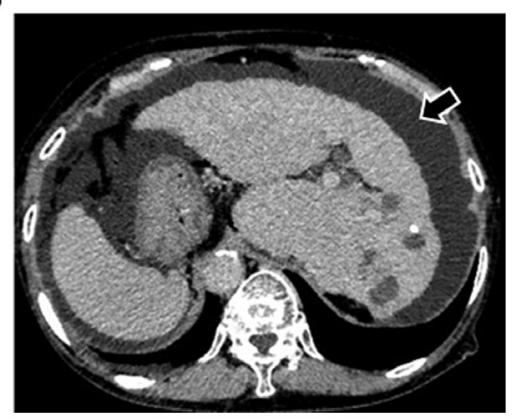

Figure 2. Representative CT images of a patient with visceral inversion administered with RAM. (A) CT image before administration of RAM. No ascites are seen. (B) CT image 27 days after administration of RAM. Severe ascites are seen (arrow). CT, computed tomography; RAM, ramucirumab.

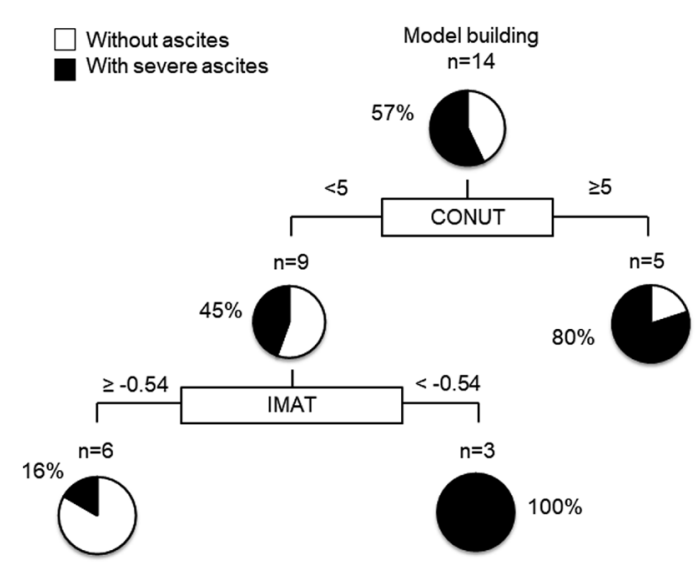

Figure 3. Profiles associated with the development of severe ascites in patients with hepatocellular carcinoma treated with RAM. Decision-tree algorithm for the development of RAM-associated severe ascites. The pie graphs indicate the percentage of patients without severe ascites (white) or with severe ascites (black) in each group. RAM, ramucirumab; CONUT, Controlling Nutritional Status; IMAT, intramuscular adipose tissue. 


\section{Discussion}

In this study, we found a high incidence of severe ascites in patients with HCC treated with RAM. Moreover, we revealed that the development of RAM-related severe ascites was associated with a profile characterized by a CONUT score $\geq 5$ and an IMAT $<-0.54$.

Our results showed a high incidence of severe ascites after treatment with RAM, even in patients with preserved liver function. RAM inhibits ligand activation of VEGF receptor 2, which promotes blood vessel permeability (25) Thus, RAM is theoretically supposed to inhibit the development of severe ascites. In fact, severe ascites has not been reported in patients with advanced gastric cancer or colon cancer treated with RAM $(26,27)$ Moreover, RAM had acceptable tolerability and safety in patients with preserved liver function in phase 3 clinical trial. Thus, our findings were different from those of previous studies. The mechanism underlying the development of severe ascites in HCC patients treated with RAM remains unclear.

In this study, the CONUT score was identified as the initial spread variable for the development of RAM-related severe ascites. A CONUT score $\geq 5$ was associated with the development of RAM-related severe ascites. A CONUT score $\geq 5$ is classified as moderate to severe malnutrition (28) Malnutrition has been associated with the development of severe ascites (29) The CONUT score is based on the total lymphocyte count, total cholesterol level, and serum albumin level. All three parameters have been reported to be associated with ascites $(30,31)$ Therefore, the CONUT score may be associated with the development of severe ascites by reflecting malnutritional status.

In patients with a CONUT score $<5$, IMAT content was selected as the variable for the second split associated with the development of RAM-related severe ascites. The prevalence of severe ascites was higher in patients with lower IMAT content. The findings suggest that the development of severe ascites was associated with low-fat infiltration of muscles. Our findings were different from a previous report that suggested that low-fat infiltration of muscles was an independent negative predictor of postoperative complications in patients with HCC (32) The reason for the association of low-fat muscle infiltration with RAM-related severe ascites in this study remains unclear. However, Addison et al reported that lower IMAT content was related to a decrease in muscle capillarization in older adults (33) Additionally, Solomon et al reported that impairment of muscle capillarization was associated with high plasma nitric oxide levels in older adults (34) Higher plasma nitric oxide levels are known to be important for the development of ascites $(35,36)$ Therefore, a lower IMAT content may be associated with the development of severe ascites through the impairment of muscle capillarization and the subsequent increase in nitric oxide production.

This study has several limitations. First, this was a retrospective study with small sample size. Second, the observational period was short. Third, no information was available on factors associated with nutritional status and IMAT content, including energy intake and physical activity. Thus, further multicenter prospective studies with large sample sizes, longer observational periods, and including lifestyle information are warranted.
In conclusion, a high incidence of severe ascites was seen in patients treated with RAM. Moreover, the development of severe ascites was associated with a CONUT score $\geq 5$ and IMAT <-0.54. Accordingly, we must be cautious of severe ascites in patients with HCC treated with RAM, in particular patients with malnutrition and muscle fatty infiltration.

\section{Acknowledgements}

Not applicable.

\section{Funding}

No funding was received.

\section{Availability of data and materials}

The datasets used and/or analyzed during the current study are available from the corresponding author on reasonable request.

\section{Authors' contributions}

NK and SS participated in the conception and design of the study, acquisition and interpretation of data, and drafting of the manuscript. KH, SK, HI, TN, TS, MN and RH participated in the acquisition of data. TK participated in the analysis and interpretation of data, and drafting of the manuscript. HM, KN, HK and TT participated in the conception, design and critical revision of the study. NK and SS confirm the authenticity of all the raw data. All authors read and approved the final manuscript.

\section{Ethics approval and consent to participate}

The present study was approved by the Ethical Committee of Kurume University School of Medicine (approval no. 19203; Kurume, Japan), and an opt-out approach was used to obtain informed consent from the patients.

\section{Patient consent for publication}

An opt-out approach was used to obtain patient informed consent for publication.

\section{Competing interests}

TK received an honorarium (lecture fee) from Mitsubishi Tanabe Pharma Corporation and Otsuka Pharmaceutical Co., Ltd. All other authors declare that they have no competing interests.

\section{References}

1. Global Burden of Disease Liver Cancer C; Akinyemiju T, Abera S, Ahmed M, Alam N, Alemayohu MA, Allen C, Al-Raddadi R, Alvis-Guzman N, Amoako Y, et al: The burden of primary liver cancer and underlying etiologies from 1990 to 2015 at the Global, Regional, and National Level: Results from the global burden of disease study 2015. JAMA Oncol 3: 1683-1691, 2017.

2. European Association for the study of the liver. Electronic address easloffice@easloffice.eu; European association for the study of the liver: EASL clinical practice guidelines: Management of hepatocellular carcinoma. J Hepatol 69: 182-236, 2018. 
3. Kudo M, Finn RS, Qin S, Han KH, Ikeda K, Piscaglia F, Baron A, Park JW, Han G, Jassem J, et al: Lenvatinib versus sorafenib in first-line treatment of patients with unresectable hepatocellular carcinoma: A randomised phase 3 non-inferiority trial. Lancet 391: 1163-1173, 2018.

4. Bruix J, Qin S, Merle P, Granito A, Huang YH, Bodoky G, Pracht M, Yokosuka O, Rosmorduc O, Breder V, et al: Regorafenib for patients with hepatocellular carcinoma who progressed on sorafenib treatment (RESORCE): A randomised, double-blind, placebo-controlled, phase 3 trial. Lancet 389: 56-66, 2017.

5. Llovet JM, Ricci S, Mazzaferro V, Hilgard P, Gane E, Blanc JF, de Oliveira AC, Santoro A, Raoul JL, Forner A, et al: Sorafenib in advanced hepatocellular carcinoma. N Engl J Med 359: 378-390, 2008

6. Zhu AX, Kang YK, Yen CJ, Finn RS, Galle PR, Llovet JM, Assenat E, Brandi G, Pracht M,Lim HY, et al: Ramucirumab after sorafenib in patients with advanced hepatocellular carcinoma and increased $\alpha$-fetoprotein concentrations (REACH-2): A randomised, double-blind, placebo-controlled, phase 3 trial Lancet Oncol 20: 282-296, 2019

7. Zhu AX, Baron AD, Malfertheiner P, Kudo M, Kawazoe S, Pezet D, Weissinger F, Brandi G, Barone CA, Okusaka T, et al: Ramucirumab as second-line treatment in patients with advanced hepatocellular carcinoma: Analysis of REACH trial results by child-pugh score. JAMA Oncol 3: 235-243, 2017.

8. Cheung K, Lee SS and Raman M: Prevalence and mechanisms of malnutrition in patients with advanced liver disease, and nutrition management strategies. Clin Gastroenterol Hepatol 10: 117-125, 2012.

9. Shimose S, Kawaguchi T, Iwamoto H, Tanaka M, Miyazaki K, Ono M, Niizeki T, Shirono T, Okamura S, Nakano M, et al Controlling nutritional status (CONUT) score is associated with overall survival in patients with unresectable hepatocellular carcinoma treated with lenvatinib: A multicenter cohort study. Nutrients 12: 1076, 2020.

10. Chavez-Tostado M, Cervantes-Guevara G, Lopez-Alvarado SE, Cervantes-Pérez G, Barbosa-Camacho FJ, Fuentes-Orozco C, Hernández-CoronaDM,González-HerediaT,Cervantes-CardonaGA and González-Ojeda A: Comparison of nutritional screening tools to assess nutritional risk and predict clinical outcomes in Mexican patients with digestive diseases. BMC Gastroenterol 20: 79, 2020.

11. Takagi K, Umeda Y, Yoshida R, Nobuoka D, Kuise T, Fushimi T, Fujiwara T and Yagi T: Preoperative controlling nutritional status score predicts mortality after hepatectomy for hepatocellular carcinoma. Dig Surg 36: 226-232, 2019.

12. Ogawa M, Lester R, Akima H and Gorgey AS: Quantification of intermuscular and intramuscular adipose tissue using magnetic resonance imaging after neurodegenerative disorders. Neural Regen Res 12: 2100-2105, 2017.

13. Fujiwara N, Nakagawa H, Kudo Y, Tateishi R, Taguri M, Watadani T, Nakagomi R, Kondo M, Nakatsuka T, Minami T, et al: Sarcopenia, intramuscular fat deposition, and visceral adiposity independently predict the outcomes of hepatocellular carcinoma. J Hepatol 63: 131-140, 2015.

14. Harimoto N, Hoshino H, Muranushi R, Hagiwara K, Yamanaka T, Ishii N, Tsukagoshi M, Igarashi T, Watanabe A, Kubo N, et al: Skeletal muscle volume and intramuscular adipose tissue are prognostic predictors of postoperative complications after hepatic resection. Anticancer Res 38: 4933-4939, 2018.

15. Forner A, Reig M and Bruix J: Hepatocellular carcinoma. Lancet 391: 1301-1314, 2018

16. Johnson PJ, Berhane S, Kagebayashi C, Satomura S, Teng M, Reeves HL, O'Beirne J, Fox R, Skowronska A, Palmer D, et al: Assessment of liver function in patients with hepatocellular carcinoma: A new evidence-based approach-the ALBI grade. J Clin Oncol 33: 550-558, 2015.

17. Koya S, Kawaguchi T, Hashida R, Hirota K, Bekki M, Goto E, Yamada M, Sugimoto M, Hayashi S, Goshima N, et al: Effects of in-hospital exercise on sarcopenia in hepatoma patients who underwent transcatheter arterial chemoembolization. J Gastroenterol Hepatol 34: 580-588, 2019.

18. Sinclair M, Gow PJ, Grossmann M and Angus PW: Review article: Sarcopenia in cirrhosis-aetiology, implications and potential therapeutic interventions. Aliment Pharmacol Ther 43: 765-777, 2016.

19. Schneider CA, Rasband WS and Eliceiri KW: NIH Image to ImageJ: 25 years of image analysis. Nat Methods 9: 671-675, 2012

20. Hirota K, Kawaguchi T, Koya S, Nagamatsu A, Tomita M, Hashida R, Nakano D, Niizeki T, Matsuse H, Shiba N and Torimura T: Clinical utility of the liver frailty index for predicting muscle atrophy in chronic liver disease patients with hepatocellular carcinoma. Hepatol Res 50: 330-341, 2020.
21. Kitajima Y, Takahashi H, Akiyama T, Murayama K, Iwane S, Kuwashiro T, Tanaka K, Kawazoe S, Ono N, Eguchi T, et al: Supplementation with branched-chain amino acids ameliorates hypoalbuminemia, prevents sarcopenia, and reduces fat accumulation in the skeletal muscles of patients with liver cirrhosis. J Gastroenterol 53: 427-437, 2018.

22. Hamaguchi Y, Kaido T, Okumura S, Kobayashi A, Shirai H, Yao S, Yagi S, Kamo N, Seo S, Taura K, et al: Preoperative visceral adiposity and muscularity predict poor outcomes after hepatectomy for hepatocellular carcinoma. Liver Cancer 8: 92-109, 2019

23. Lencioni R and Llovet JM: Modified RECIST (mRECIST) assessment for hepatocellular carcinoma. Semin Liver Dis 30: $52-60,2010$.

24. Shimose S, Tanaka M, Iwamoto $H$, Niizeki T, Shirono $T$, Aino H, Noda Y, Kamachi N, Okamura S, Nakano M, et al: Prognostic impact of transcatheter arterial chemoembolization (TACE) combined with radiofrequency ablation in patients with unresectable hepatocellular carcinoma: Comparison with TACE alone using decision-tree analysis after propensity score matching. Hepatol Res 49: 919-928, 2019.

25. Yoshiji H, Kuriyama S, Hicklin DJ, Huber J, Yoshii J, Ikenaka Y, Noguchi R, Nakatani T, Tsujinoue $\mathrm{H}$ and Fukui H: The vascular endothelial growth factor receptor KDR/Flk-1 is a major regulator of malignant ascites formation in the mouse hepatocellular carcinoma model. Hepatology 33: 841-847, 2001.

26. Tabernero J, Takayuki Y and Cohn AL: Correction to Lancet Oncol 2015; 16: 499-508. Ramucirumab versus placebo in combination with second-line FOLFIRI in patients with metastatic colorectal carcinoma that progressed during or after first-line therapy with bevacizumab, oxaliplatin, and a fluoropyrimidine (RAISE): A randomised, double-blind, multicentre, phase 3 study. Lancet Oncol 16: e262, 2015.

27. Wilke H, Muro K, Van Cutsem E, Oh SC, Bodoky G, Shimada Y, Hironaka S, Sugimoto N, Lipatov O, Kim TY, et al: Ramucirumab plus paclitaxel versus placebo plus paclitaxel in patients with previously treated advanced gastric or gastro-oesophageal junction adenocarcinoma (RAINBOW): A double-blind, randomised phase 3 trial. Lancet Oncol 15: 1224-1235, 2014.

28. Saito A, Amiya E, Hatano M, Shiraishi Y, Nitta D, Minatsuki S, Maki H, Hosoya Y, Tsuji M, Bujo C, et al: Controlling nutritional status score as a predictive marker for patients with implantable left ventricular assist device. ASAIO J 66: 166-172, 2020.

29. Vidot H, Bowen DG, Carey S, McCaughan GW, Allman-Farinelli M and Shackel NA: Aggressive nutrition intervention reduces ascites and frequency of paracentesis in malnourished patients with cirrhosis and ascites. JGH Open 1: 92-97, 2017.

30. Bernardi M, Angeli P, Claria J, Moreau R, Gines P, Jalan R, Caraceni P, Fernandez J, Gerbes AL, O'Brien AJ, et al: Albumin in decompensated cirrhosis: New concepts and perspectives. Gut 69: 1127-1138, 2020.

31. Zhao LY, Yang DD, Ma XK, Liu MM, Wu DH, Zhang XP, Ruan DY, Lin JX, Wen JY, Chen J, et al: The prognostic value of aspartate aminotransferase to lymphocyte ratio and systemic immune-inflammation index for overall survival of hepatocellular carcinoma patients treated with palliative treatments. J Cancer 10: 2299-2311, 2019.

32. Hamaguchi Y, Kaido T, Okumura S, Kobayashi A, Fujimoto Y, Ogawa K, Mori A, Hammad A, Hatano E and Uemoto S: Muscle steatosis is an independent predictor of postoperative complications in patients with hepatocellular carcinoma. World J Surg 40: 1959-1968, 2016.

33. Addison O, Ryan AS, Blumenthal J and Prior SJ: Increased intramuscular adipose tissue is related to increased capillarization in older adults. J Frailty Aging 9: 134-138, 2020.

34. Solomon TP, Haus JM, Li Y and Kirwan JP: Progressive hyperglycemia across the glucose tolerance continuum in older obese adults is related to skeletal muscle capillarization and nitric oxide bioavailability. J Clin Endocrinol Metab 96: 1377-1384, 2011.

35. Ferguson JW, Dover AR, Chia S, Cruden NL, Hayes PC and Newby DE: Inducible nitric oxide synthase activity contributes to the regulation of peripheral vascular tone in patients with cirrhosis and ascites. Gut 55: 542-546, 2006.

36. AngeliP,Fernandez-Varo G, Dalla Libera V,Fasolato S, Galioto A, Arroyo V, Sticca A, Guarda S, Gatta A and Jiménez W: The role of nitric oxide in the pathogenesis of systemic and splanchnic vasodilation in cirrhotic rats before and after the onset of ascites. Liver Int 25: 429-437, 2005.

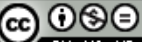

This work is licensed under a Creative Commons Attribution-NonCommercial-NoDerivatives 4.0 International (CC BY-NC-ND 4.0) License. 